\title{
A SOBOLEV INEQUALITY FOR PLURIHARMONIC FUNCTIONS
}

\author{
STEVEN R. BELL ${ }^{1}$
}

\begin{abstract}
A Sobolev inequality is proved which implies that, on a smooth bounded domain $D$ contained in $\mathbf{C}^{n}$, the $L^{2}$ inner product of two pluriharmonic functions is defined whenever one of them is in $C^{\infty}(\bar{D})$ and the other is dominated by some negative power of the distance to the boundary.
\end{abstract}

Suppose $D$ is a smooth bounded domain in $\mathbf{C}^{n}$. We shall prove that for each positive integer $s$, there is a constant $C=C(s)$ such that

$$
\left|\int_{D} f g\right| \leq C\|f\|_{s}\|g\|_{-s}
$$

for all pluriharmonic functions $f$ and $g$ in $L^{2}(D)$. Here $\|f\|_{s}^{2}=\sum_{|\alpha| \leq s} \int_{D}\left|D^{\alpha} f\right|^{2}$ is the usual Sobolev $s$-norm and

$$
\|g\|_{-s}=\operatorname{Sup}\left\{\left|\int_{D} g \phi\right|: \phi \in C_{0}^{\infty}(D) ;\|\phi\|_{s}=1\right\} .
$$

This Sobolev inequality implies that for each positive integer $s$, there is an integer $m=m(s)$ and a constant $K=K(s)$ such that for pluriharmonic functions $f$ and $g$ in $L^{2}(D)$,

$$
\left|\int_{D} f g\right| \leq K\left(\operatorname{Sup}_{\substack{|\alpha| \leq m \\ z \in D}}\left|D^{\alpha} f(z)\right|\right)\left(\operatorname{Sup}_{z \in D}|g(z)| d(z)^{s}\right)
$$

where $d(z)$ is equal to the distance of $z$ to $b D$, the boundary of $D$. Inequality (1.2) is a simple consequence of $(1.1)$ because $\|g\|_{-s-n-1} \leq c \operatorname{Sup}\left\{|g(z)| d(z)^{s}: z \in D\right\}$ for pluriharmonic functions $g$ (see [2]).

Inequality (1.1) is known to hold for holomorphic functions $f$ and $g$ (see [1]) and has proved to be very useful in the study of boundary behavior of holomorphic mappings. It is the purpose of this note to extend (1.1) to pluriharmonic functions.

Let $W^{s}(D)$ denote the usual Sobolev space of complex valued functions on $D$ with derivatives up to order $s$ in $L^{2}(D)$. Let $W_{0}^{s}(D)$ be the closure of $C_{0}^{\infty}(D)$ in $W^{s}(D)$ and let $P^{s}(D)$ be the subspace of $W^{s}(D)$ consisting of pluriharmonic functions. A function $u$ is said to vanish to order $t$ on $b D$ if $D^{\alpha} u(z)=0$ for all multi-indices $\alpha$ with $|\alpha| \leq t$ and all $z \in b D$. It is easy to verify that a function in $C^{t}(\bar{D})$ which vanishes to order $t-1$ on $b D$ is in $W_{0}^{t}(D)$.

We shall prove (1.1) by constructing a linear operator $L^{s}$ which is bounded from $P^{s}(D)$ to $W_{0}^{s}(D)$ such that $\int_{D} f g=\int_{D}\left(L^{s} f\right) g$ for all pluriharmonic functions $g$ in $L^{2}(D)$. Then (1.1) follows because when $f$ and $g$ are pluriharmonic and in $L^{2}(D)$,

Received by the editors February 13, 1981.

1980 Mathematics Subject Classification. Primary 32A99; Secondary 32H99.

${ }^{1}$ Research supported by NSF grant \#MCS 80-17205. 
we obtain

$$
\left|\int_{D} f g\right|=\left|\int_{D}\left(L^{s} f\right) g\right| \leq\left\|L^{s} f\right\|_{s}\|g\|_{-s} \leq C\|f\|_{s}\|g\|_{-s} .
$$

Construction of the operators $L^{s}$. Let $r$ be a $C^{\infty}$ function such that $D=$ $\{r<0\}, b D=\{r=0\}$ and $d r \neq 0$ on $b D$. For $u \in C^{\infty}(\bar{D})$, we set

$$
L^{s} u=u-\Delta\left(\sum_{k=0}^{s-1} \theta_{k} r^{k+2}\right)
$$

where the functions $\theta_{k}$ are to be determined inductively. Notice that the function acted upon by the Laplacian in (1.3) vanishes to second order on $b D$, and therefore that $\int_{D} u g=\int_{D}\left(L^{s} u\right) g$ when $g$ is pluriharmonic by integration by parts.

Let $X$ be a $C^{\infty}$ function which is equal to 1 in a neighborhood of $b D$ and zero in a neighborhood of $\{|\nabla r|=0\}$. Define $L^{1} u=u-\Delta\left(\theta_{0} r^{2}\right)$ where $\theta_{0}=\frac{1}{2} X|\nabla r|^{-2} u$. The function $\theta_{0}$ has been chosen in this way so that $L^{1} u$ vanishes on $b D$, i.e., so that $L^{1} u \in W_{0}^{1}(D)$.

Suppose $\theta_{0}, \theta_{1}, \ldots, \theta_{t-1}$ have been chosen so that $L^{t} u$ vanishes to order $t-1$ on $b D$. We set $L^{t+1} u=L^{t} u-\Delta\left(\theta_{t} r^{t+2}\right)$ where $\theta_{t}$ is to be determined so that $L^{t+1} u$ vanishes to order $t$ on $b D$. Note that no matter what we choose $\theta_{t}$ to be, $L^{t+1} u$ vanishes to order $t-1$ on $b D$. Let $(\partial / \partial n)$ be a vector field which points in the normal direction on $b D$. For convenience, let us take $(\partial / \partial n)=(\nabla r \cdot \nabla) /|\nabla r|^{2}$. We set

$$
\theta_{t}=\frac{X}{(t+2) !}|\nabla r|^{-2}\left(\frac{\partial}{\partial n}\right)^{t} L^{t} u
$$

so that $(\partial / \partial n)^{t} L^{t+1} u=0$ on $b D$. This guarantees that $L^{t+1} u$ vanishes to order $t$ on $b D$ and hence that $L^{t+1} u \in W_{0}^{t+1}(D)$. The induction is complete.

To finish the proof, we must show that $L^{s}$ is bounded from $P^{s}(D)$ to $W_{0}^{s}(D)$. We shall use the shorthand notation:

$$
\partial^{\alpha} \bar{\partial}^{\beta}=\left(\frac{\partial^{\alpha}}{\partial z^{\alpha}}\right)\left(\frac{\partial^{\beta}}{\partial \bar{z}^{\beta}}\right) .
$$

A simple induction reveals that $L^{s}$ is a linear differential operator of the form

$$
\sum_{|\alpha|+|\beta| \leq k \leq N_{0}} A_{\alpha, \beta, k} r^{k} \partial^{\alpha} \bar{\partial}^{\beta}
$$

where $N_{s}=\frac{s}{2}(s+3)$ and $A_{\alpha, \beta, k} \in C^{\infty}(\bar{D})$. We now prove that any operator of the form (1.4) maps $P^{s}(D)$ to $W^{s}(D)$ boundedly. If $f$ is a pluriharmonic function in $C^{\infty}(\bar{D}),|\alpha|+|\beta| \leq k, A \in C^{\infty}(\bar{D})$, and $D^{\gamma}$ is a derivative of order $|\gamma| \leq s$, then

$$
\begin{aligned}
\int_{D}\left|D^{\gamma}\left(A r^{k} \partial^{\alpha} \bar{\partial}^{\beta} f\right)\right|^{2} & =\sum_{|\omega|+|\delta|+m=2|\gamma|} \int_{D} A_{\omega, \delta} r^{2 k-m} D^{\omega} \partial^{\alpha} \bar{\partial}^{\beta} f D^{\delta} \bar{\partial}^{\alpha} \partial^{\beta} \bar{f} \\
& =\sum_{\substack{|\omega| \leq|\gamma| \\
|\delta| \leq|\gamma|}} \int_{D} \tilde{A}_{\omega, \delta} D^{\omega} f D^{\delta} \bar{f} \leq C\|f\|_{s}^{2} .
\end{aligned}
$$

Here we have used integration by parts and the fact that $\partial^{\rho} \bar{\partial}^{\rho} f=0$ for any multiindex $\rho$. The power of $r$ in the integrand is exactly large enough so that derivatives 
from $\partial^{\alpha} \bar{\partial}^{\beta}$ can be taken off $f$ via integration by parts until there are $|\gamma|$ or less derivatives remaining.

We have now obtained that there is a constant $C$ such that $\left\|L^{s} f\right\|_{s} \leq C\|f\|_{s}$ for pluriharmonic functions $f$ in $C^{\infty}(\bar{D})$. It remains for us to prove that this inequality also holds for a general function $f$ in $P^{s}(D)$. To do this, we shall approximate $f$ in $W^{s}(D)$ by functions of the form $f_{\epsilon}(z)=\sum_{j=0}^{m} \phi_{j} f\left(z-\epsilon \nu_{j}\right)$ where $\epsilon$ is a small number greater than zero, $\left\{\nu_{j}\right\}_{j=1}^{m}$ is a set of unit vectors in $\mathbf{C}^{n}$, and $\left\{\phi_{j}\right\}_{j=0}^{m}$ is a partition of unity of $\bar{D}$ such that $\phi_{0} \in C_{0}^{\infty}(D)$ and $\nu_{0}=0$, and such that for $j \geq 1$, Supp $\phi_{j}$ intersects $b D$ in a small enough set so that the unit vector $\nu_{j}$ points out of $D$ and intersects $b D$ transversally at all points in a neighborhood of Supp $\phi_{j} \cap b D$. The partition $\left\{\phi_{j}\right\}$ and the vectors $\left\{\nu_{j}\right\}$ can be chosen independently of $f$. Note that $L^{s} f_{\epsilon}$ can be written in the form

$$
\sum_{j=1}^{m} \sum_{|\alpha|+|\beta| \leq k \leq N_{s}} A_{\alpha, \beta, k}^{j} r^{k} \partial^{\alpha} \bar{\partial}^{\beta} f\left(z-\epsilon \nu_{j}\right)
$$

where the functions $A_{\alpha, \beta, k}^{j}$ have support contained in Supp $\phi_{j}$, are in $C^{\infty}(\bar{D})$, and do not depend on $f$. Now the Sobolev $s$-norm of each term

$$
A_{\alpha, \beta, k}^{j} r^{k} \partial^{\alpha} \bar{\partial}^{\beta} f\left(\cdot-\epsilon \nu_{j}\right)
$$

can be estimated exactly as before since $f\left(\cdot-\epsilon \nu_{j}\right)$ is pluriharmonic in a neighborhood of Supp $\phi_{j} \cap \bar{D}$. This procedure yields the estimate,

$$
\left\|L^{s} f_{\epsilon}\right\|_{s} \leq C \sum\left\|f\left(\cdot-\epsilon \nu_{j}\right)\right\|_{W^{s}\left(\operatorname{Supp} \phi_{j} \cap D\right)} \leq m C\|f\|_{s} .
$$

Hence, for small $\epsilon>0$, the functions $L^{s} f_{\epsilon}$ form a bounded set in $W^{s}(D)$. The Banach-Saks theorem [3, p. 181] implies that there is a sequence of numbers $\epsilon_{i}>0$ with $\epsilon_{i} \rightarrow 0$ as $i \rightarrow \infty$ such that the arithmetic means of the sequence $\left\{L^{s} f_{\epsilon_{i}}\right\}_{i=1}^{\infty}$ converge in $W^{s}(D)$ to some function $v$ in $W^{s}(D)$. It is clear that $v=L^{s} f$, and therefore that $\left\|L^{s} f\right\|_{s} \leq m C\|f\|_{s}$. This completes the proof.

REMARK. The inequality (1.1) suggests the definition of a special norm on pluriharmonic functions given by

$$
\|\| f \|_{s}=\operatorname{Sup}\left\{\left|\int_{D} f g\right|:\|g\|_{-s}=1 ; g \in P(D)\right\},
$$

where $P(D)$ denotes the space of $L^{2}$ pluriharmonic functions. It is conceivable that the norm $\||f|\|_{s}$ is equivalent to the norm $\|f\|_{s}$ for $f$ in $P^{s}(D)$. However, a proof of this fact requires detailed knowledge of the regularity properties of the operator which projects $L^{2}(D)$ onto its subspace $P(D)$ consisting of pluriharmonic functions.

\section{REFERENCES}

1. S. Bell, Biholomorphic mappings and the $\bar{\partial}$-problem, Ann. of Math. (2) 114 (1981), 103-113.

2. - Proper holomorphic mappings and the Bergman projection, Duke Math. J. 48 (1981), 167175 .

3. L. Bers, F. John and M. Schechter, Partial differential equations, Lectures in Appl. Math., Proceedings of the Summer Seminar (Boulder, Colorado, 1957), Vol. 3A, Amer. Math. Soc., Providence, R. I., 1979.

Department of Mathematics, Princeton University, Princeton, NeW Jersey 